\title{
Single-Layer Wideband Circularly Polarized High-Efficiency Reflectarray for Satellite Communications
}

\author{
Long Zhang, Steven Gao, Senior Member, IEEE, Qi Luo, Member, IEEE, Wenting Li, and Qingxia Li, \\ Member, IEEE
}

\begin{abstract}
This paper presents a single-layer circularly polarized $(\mathrm{CP})$ reflectarray which achieves large bandwidth in terms of axial ratio (AR), gain, aperture efficiency and radiation pattern simultaneously. By using a novel wideband $S$-shaped phasing element, an offset-fed reflectarray with 200 offset beam is designed based on the element angular rotation method. Theoretical analysis is given to analyze the effect of angular rotated elements on the performance of the reflectarray, which indicates that the AR bandwidth of the reflectarray can exceed the AR bandwidth of the feed horn. Besides, the effect of the differential spatial phase delay on the bandwidth of the proposed reflectarray is analyzed quantitatively. To verify these concepts, a $180 \mathrm{~mm} \times 180 \mathrm{~mm}$ prototype with $15 \times 15$ elements is fabricated and measured. The measured results confirm that the proposed reflectarray can achieve a $68.5 \% 3 \mathrm{~dB}$ AR bandwidth and a $47.8 \%$ $3 \mathrm{~dB}$ gain bandwidth. Moreover, the aperture efficiency is larger than $50 \%$ in a $33 \%$ bandwidth and larger than $30 \%$ in a $64 \%$ bandwidth.
\end{abstract}

Index Terms-Circular polarization, wideband array, element rotation method, reflectarray,

\section{INTRODUCTION}

$\mathrm{P}$ RINTED reflectarrays combine the advantages of parabolic antennas and planar phased arrays, which receive increasing interest in designing high-gain antennas. Generally, reflectarrays consist of radiating elements with preadjusted phases and an illuminating feed antenna to form a planar phase front in the farfield [1]. Although they have many advantages, such as the significantly simplified feeding system, versatile beams, flat structure and low manufacturing cost, one major drawback of reflectarrays is their narrow bandwidth.

The narrow bandwidth of reflectarrays is mainly caused by two factors: the inherent narrow bandwidth of microstrip elements and the differential spatial phase delay caused by different path lengths from the feed to each element [2]. To improve the bandwidth of reflectarrays, several approaches were adopted, such as using stacked patches [3], subwavelength element [4], dual-frequency phase synthesis [5] and true time delay technique [6].

Manuscript received December XX, 2016.

L. Zhang, S. Gao, Q. Luo and W. Li are with the School of Engineering an d Digital Arts, University of Kent, Canterbury CT2 7NT, UK. (emails: 1z76@ kent.ac.uk; s.gao@kent.ac.uk).
On the other hand, designing circularly polarized (CP) reflectarray is necessary for some applications since the $\mathrm{CP}$ arrays possess the advantages of mitigation of multi-path fading, immunity of "Faraday rotation" and the reduction of polarization mismatching [7]. Two different ways have been introduced to design a CP reflectarray. The first one is using a linearly polarized (LP) feed to illuminate an aperture which is able to transform the incident LP wave to CP reflected wave and form a cophasal beam in the far-field simultaneously [8, 9]. Another method to design a CP reflectarray is utilizing a $\mathrm{CP}$ feed as a primary source and the reflecting surface can reflect the incident CP wave directly and yields a planar phase front in the far-field. Various designs based on this approach were proposed, including using angular rotation elements [10, 11], variable sized elements [12] and elements with variable-length phase delay lines [13].

Considering that it is more difficult to design elements with wideband $\mathrm{CP}$ reflection performance and wideband $\mathrm{CP}$ feeds, the bandwidth enhancement of $\mathrm{CP}$ reflectarrays is thus more challenging than LP reflectarrays. To meet frequency coverage requirement, designing dual-frequency $\mathrm{CP}$ reflectarrays is attractive to some applications [14, 15]. However, for applications such as high data rate satellite communications, it is highly desirable to achieve CP reflectarrays with broad bandwidth [16]. Different approaches were utilized to improve the bandwidth of CP reflectarrays [9, 17-20]. Through using rectangular-patch phasing element with subwavelength grid spacing, a 17\% 1-dB gain bandwidth and 11\% 3-dB axial ratio (AR) bandwidth was obtained [17]. The bandwidth of CP reflectarray was further improved by using a dual-layer $\mathrm{T}$ shaped phasing element, with a 1-dB gain bandwidth of $20 \%$ and 3-dB AR bandwidth of $28 \%$ [18]. Since the elements used in $[17,18]$ are resonant type, the CP reflectarray using these resonant phasing elements demonstrates a typically smaller than $30 \%$ bandwidth. Multi-resonance phasing elements such as the Jerusalem cross and open loop combined subwavelength element [9] and the dual-ring element [19] based CP reflectarrays achieved more than 30\% 3-dB AR bandwidth. Another way to realize wide bandwidth of CP reflectarrays is

Q. Li is with the School of Electronic Information and Communications, Huazhong University of Science and Technology, Wuhan 430074, China. 
using spatial time-delay units (TDUs) [20]. Although the CP reflectarray using TDUs achieved $40 \% 3-\mathrm{dB}$ gain bandwidth and 3-dB AR bandwidth, the aperture efficiency was just around $40 \%$ and the multi-layer structure increased the fabrication complexity.

In this paper, a novel S-shaped phasing element which achieves smaller than $-15 \mathrm{~dB}$ cross polarization (cross-pol) reflection in a 2:1 bandwidth is proposed. Based on this novel broadband element, a single-layer CP reflectarray using angular rotating elements is designed, fabricated and measured. The measured results indicate that the proposed single-layer reflectarray achieves a $68.5 \% 3 \mathrm{~dB}$ AR bandwidth and a $47.8 \%$ $3 \mathrm{~dB}$ gain bandwidth. Moreover, the aperture efficiency (AE) is larger than $50 \%$ in a $33 \%$ bandwidth and larger than $30 \%$ in a $64 \%$ bandwidth. To the best knowledge of the authors, it is the widest single-layer $\mathrm{CP}$ reflectarray developed up to now in terms of 3-dB AR bandwidth and larger than 50\% $\mathrm{AE}$ bandwidth.

The paper is organized as follows: Section II introduces the design of the novel S-shaped element; Section III presents the design and analysis of the proposed CP reflectarray; Section IV presents the simulation and measurement results and comparisons with other reported wideband $\mathrm{CP}$ reflectarrays. The conclusion is given in Section V.

\section{S-SHAPED REFLECTARRAY ELEMENT}

Various antenna elements have been designed and deployed in CP reflectarrays, such as the patches, stacked patches, crossed dipoles and their derivatives, split rings and the combination of several types of these elements. Generally, all these reported elements demonstrate a bandwidth less than $50 \%$. In this section, a novel S-shaped element is presented and analyzed, which can achieve a bandwidth over $66 \%$.

\section{A. Element Geometry}

The geometry of the proposed S-shaped element is shown in Fig. 1.

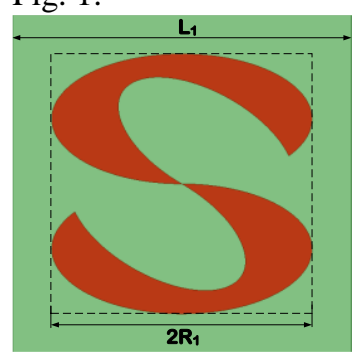

(a)

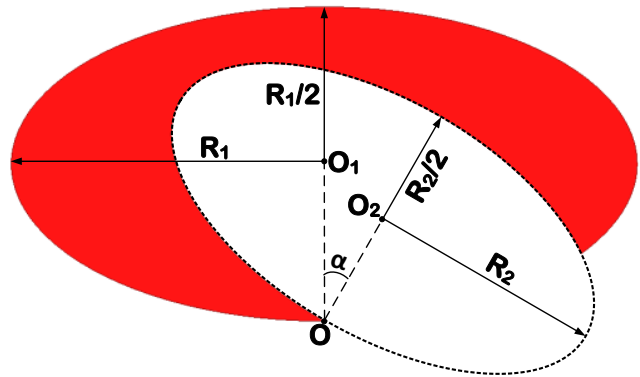

(c)

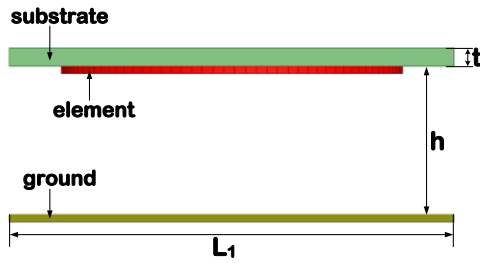

(b)

Fig. 1. Geometry of the proposed element: (a) top view, (b) side view, (c) single arm.
As shown in Fig. 1 (a) and (b), the proposed element is printed on the bottom layer of a Rogers RO4003C substrate with relative dielectric constant of 3.55 , dissipation factor of 0.0027 and a thickness of $t$. A ground plane is placed below the substrate with a height of $h$. The size of the ground plane and the unit cell are $\mathrm{L}_{1} \times \mathrm{L}_{1}$. The $\mathrm{S}$-shaped element is composed of two single arms with the same size shown in Fig. 1 (c). It can be seen from Fig. 1 (c) that each arm is obtained by subtracting a smaller sized ellipse with a rotating angle $\alpha$ from a larger sized ellipse. To form a square $\mathrm{S}$-shaped element, the major radius of the bigger ellipse $\mathrm{R}_{1}$ is two times as large as the minor radius.

The proposed element is a further study from the antenna presented in [21], where an inverted S-shaped antenna with wideband $\mathrm{CP}$ radiation was presented. The proposed phasing element has a more compact size $(0.32 \lambda \times 0.32 \lambda)$ than the antenna presented in [21] $(0.99 \lambda \times 0.42 \lambda)$. The size reduction is realized by choosing bigger rotating angle $\alpha$ and bigger $R_{1} / R_{2}$ ratio, which gives longer travelling-wave current path and the utilization of sub-wavelength technique. Although the size of the presented element is greatly reduced, the $\mathrm{CP}$ bandwidth is largely improved by $58.8 \%$ (from $42 \%$ in [21] to $66.7 \%$ ). Furthermore, the proposed element can be placed in a square lattice while the antenna in [21] is only able to be placed in a rectangular lattice. The square-sized element is more advantageous to planar arrays because the rectangular-sized antenna has different electrical length along $\mathrm{x}$-axis and $\mathrm{y}$-axis direction, which results in uneven characteristics such as different beamwidth and AR beamwidth in the two orthogonal directions. On the contrary, the proposed element can provide relatively symmetry performance along $\mathrm{x}$-axis and $\mathrm{y}$-axis direction, which brings more equal mutual coupling between each elements in the two directions and thus facilitates the design and improves the performance of the reflectarray.

The detailed geometry dimensions of the proposed element are shown in Table I.

TABLE I

ELEMENT DIMENSIONS

\begin{tabular}{|c|c|c|c|c|c|}
\hline $\mathrm{L}_{1}$ & $\mathrm{R}_{1}$ & $\mathrm{R}_{2}$ & $\alpha$ & $\mathrm{h}$ & $\mathrm{t}$ \\
\hline $12 \mathrm{~mm}$ & $4.6 \mathrm{~mm}$ & $3.45 \mathrm{~mm}$ & $30^{\circ}$ & $4 \mathrm{~mm}$ & $0.508 \mathrm{~mm}$ \\
\hline
\end{tabular}

\section{B. Element Performance}

As the electrical length of the proposed element is mainly determined by geometry parameters $R_{1}, R_{2}$ and rotating angle $\alpha$, the impact of these parameters to the element's performance needs to be investigated. For a brief investigation, the ratio $R_{2} / R_{1}=0.75$ and the rotating angle $\alpha$ is kept unchanged while only $R_{1}$ is varied.

The evaluation of the element performance is conducted by using a periodic boundary and a Floquet port excitation in HFSS. The incident field is set to be right-hand circularly polarized (RHCP). Fig. 2 shows the magnitudes of reflected RHCP field (co-pol component) and left-hand circularly polarized (LHCP) field (cross-pol component) with different $R_{1}$. As shown, the available bandwidth $\left(\left|\Gamma_{\text {cross-pol }}\right| \leq-15 \mathrm{~dB}\right)$ of the element increases with larger $R_{1}$. As the ratio $R_{2} / R_{1}$ is kept unchanged, this phenomenon can be explained that 
increasing $\mathrm{R}_{1}$ brings longer electrical length and pushes the lowest-frequency resonance to lower frequency while has little influence on higher-frequency resonance.

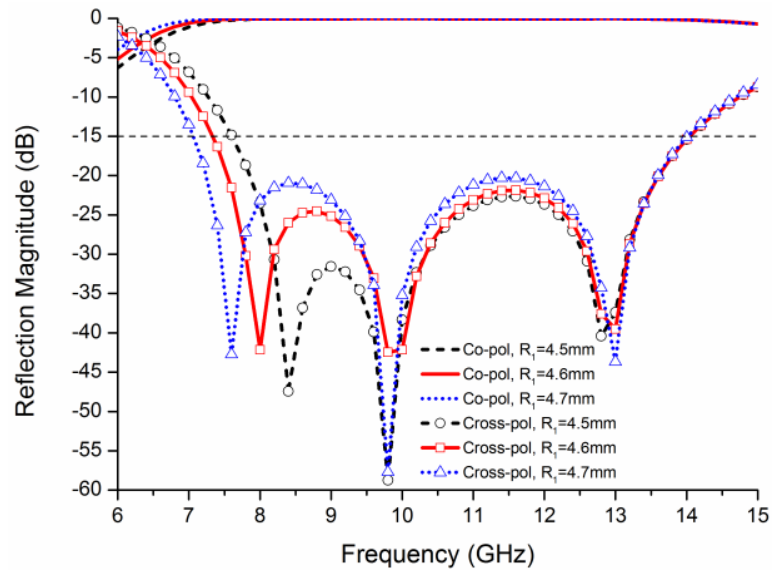

Fig. 2. The magnitude of the reflected field with different $R_{1}$.

As shown in Fig. 2, the element can provide a bandwidth of 2:1 $(7 \mathrm{GHz}$ to $14 \mathrm{GHz})$ within which the reflected LHCP component is smaller than $-15 \mathrm{~dB}\left(\left|\Gamma_{\text {cross-pol }}\right| \leq-15 \mathrm{~dB}\right)$ when $\mathrm{R}_{1}$ is $4.7 \mathrm{~mm}$. Notice that $\left|\Gamma_{\text {cross }- \text { pol }}\right|^{2}+\left|\Gamma_{\text {co-pol }}\right|^{2} \cong 1$ (the approximation rises from dielectric and conductor losses), thus $\left|\Gamma_{\text {cross-pol }}\right| \leq-15 \mathrm{~dB}$ makes $\left|\Gamma_{\text {co-pol }}\right| \geq-0.2 \mathrm{~dB}$ which indicates that the element has low-level cross-pol field reflection while keep the co-pol component nearly non-degraded. To acquire lower cross-pol component reflection and adequate bandwidth simultaneously, $R_{1}$ is chosen to be $4.6 \mathrm{~mm}$. In this case, the available bandwidth is from $7.3 \mathrm{GHz}$ to $14 \mathrm{GHz}$ and $\left|\Gamma_{\text {cross-pol }}\right|$ $\leq-22 \mathrm{~dB},\left|\Gamma_{\text {co-pol }}\right| \geq-0.08 \mathrm{~dB}$ from $7.6 \mathrm{GHz}$ to $13.5 \mathrm{GHz}$.

The element rotating method is used to provide the desired phases for each element. It is indicated that the angular rotation angle $\psi$ of a CP element results in a $2 \psi$ phase variation of the reflected co-pol field $[10,22]$. By choosing the required angular rotation angle of each element, a cophasal beam can be realized in the far-field. Fig. 3 shows the phase difference of the reflected co-pol field under different element rotation angle. As shown, the element achieves a rather linear phase response to the element rotation angle $\psi$ in a 2:1 frequency range. The phase error (actual phase difference compared with $2 \psi$ ) is smaller than $10^{\circ}$ within the bandwidth.

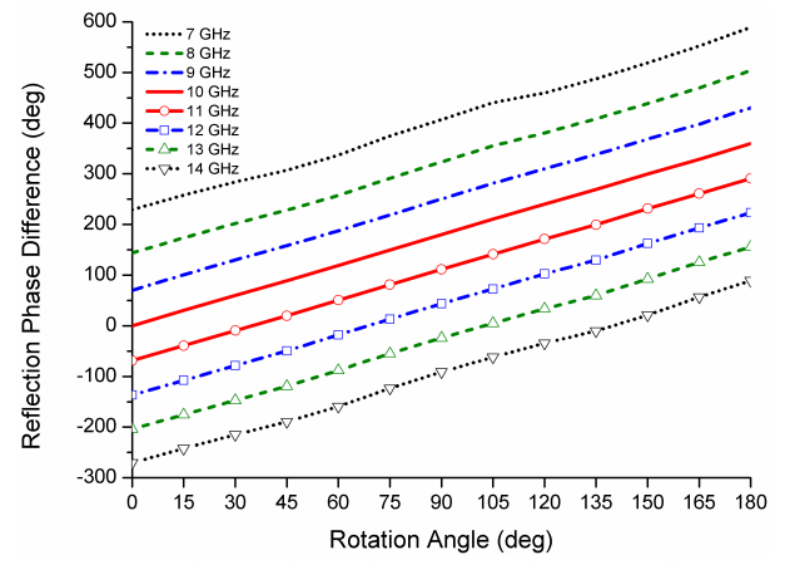

Fig. 3. The phase difference of the reflected co-pol field with different element rotation angle.
The reflection magnitudes of the co-pol and cross-pol components with different rotation angle $\psi$ are shown in Fig. 4. As shown, the proposed element maintains low cross-pol and good co-pol reflection in a nearly 2:1 bandwidth frequency range under all rotation angles. It is worthy pointing out that the proposed element exhibits the same reflection magnitude performance with the reflected LHCP field as co-pol component when the incident field changes to LHCP. In this case, the reflected phase variation of the co-pol field introduced by the element rotation angle $\psi$ is $-2 \psi$, which is different to the situation of RHCP incident field.

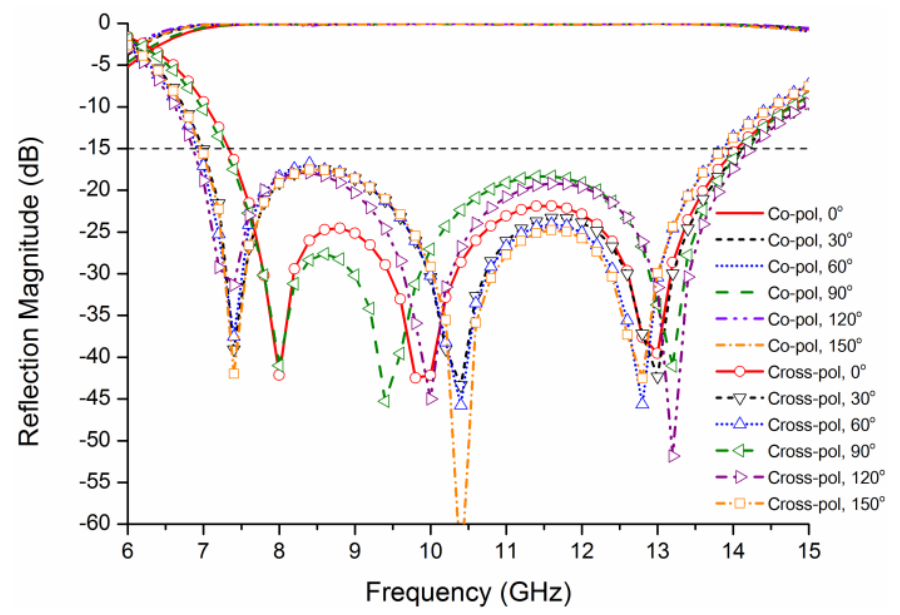

Fig. 4. The magnitude of the reflected field with different rotation angle.

\section{REFLECTARRAY DESIGN AND ANALYSIS}

In this section, the array configuration and the element phase distribution over the aperture are given at first. Then, the performance of the proposed reflectarray is analyzed theoretically and the conclusions are verified by using different feed horns. Finally, the effect of the differential spatial phase delay on the bandwidth of the proposed reflectarray is analyzed.

\section{A. Array Configuration}

The configuration of the proposed reflectarray is shown in Fig. 5. As shown, the reflectarray consists of $225(15 \times 15) \mathrm{S}$ shaped elements, each with different rotation angle to provide required phase delay. The reflectarray is designed to be fed by a wideband dual-CP horn with an offset of $20^{\circ}$ to the Z-axis. The offset configuration is mainly to minimize the blockage of the feed. It is indicated in [23] that an offset feed causes main beam scan with frequency due to a change in phase taper across the reflectarray with a change in frequency. This beam squint phenomenon can be minimized if the angle of the main beam is chosen to be close to the natural specular reflection angle. To eliminate the beam squint, the main beam direction is designed to $\theta=-20^{\circ}$. The f/D ratio is chosen to be 0.97 . 


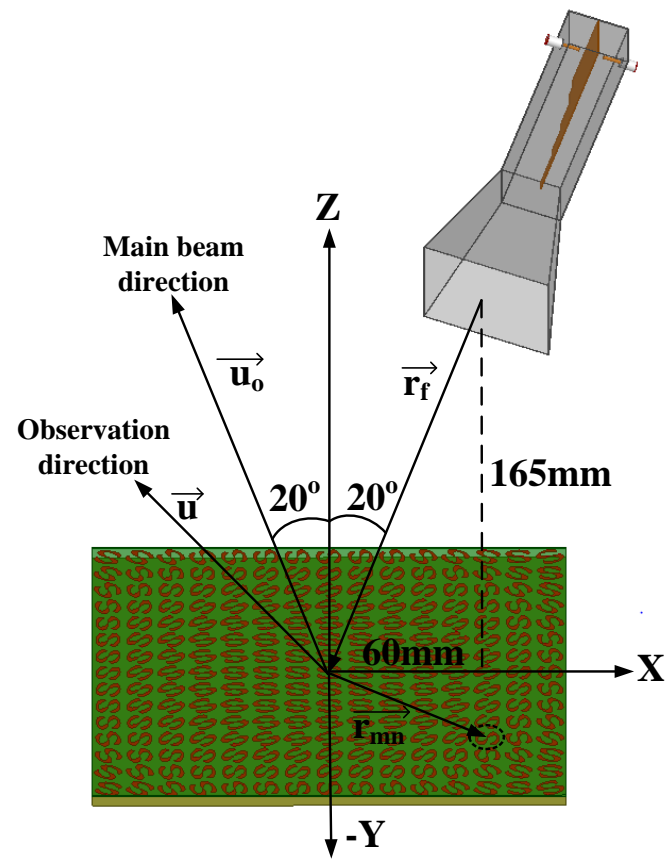

(a)

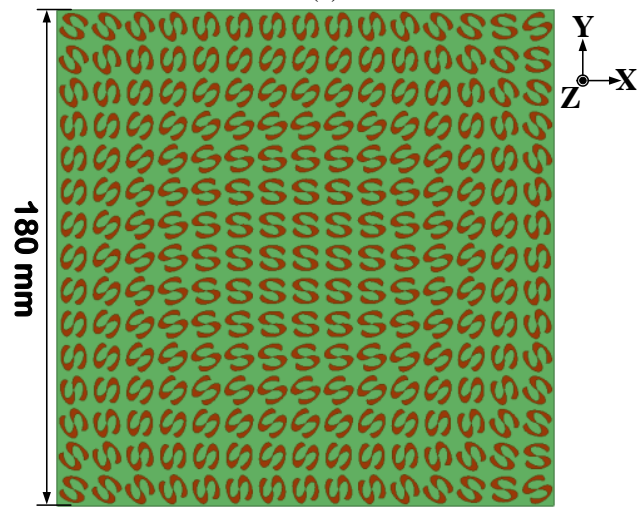

(b)

Fig. 5. Configuration of the proposed reflectarray: (a) side view, (b) top view of reflecting aperture.

The compensated phase distribution of each S-shaped element is shown in Fig. 6. As the element rotation angle $\psi$ results in a $2 \psi$ phase variation of the reflected co-pol field, the compensated phase distribution in Fig. 6 can directly map into Fig. 5 (b).

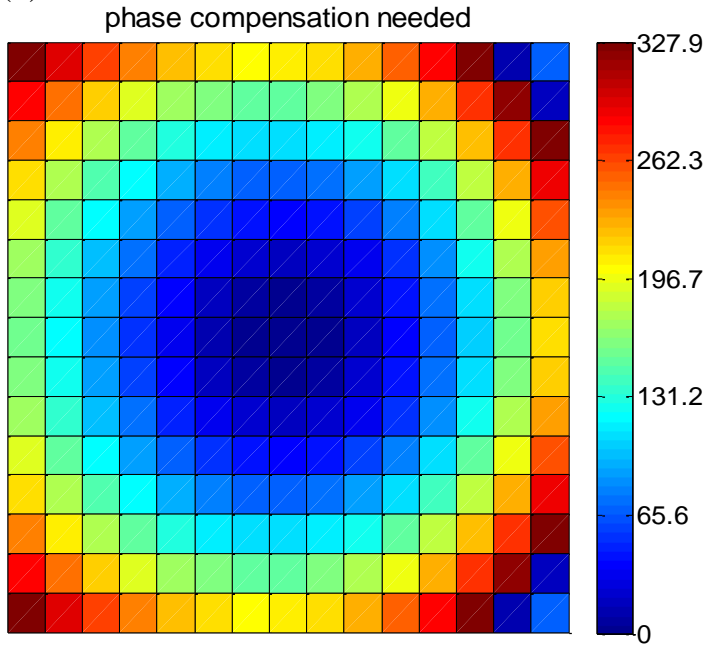

Fig. 6. Compensated phase distribution for each element.

\section{B. Analysis of Array Performance Using Angular Rotated Elements}

It is implicit that the cross-pol of a reflectarray using element rotating method is degraded in the main beam region and thus the AR performance can be enhanced [10]. However, this phenomenon is not discussed in details in [10]. Besides, the reflectarray proposed in [10] is a narrow-band array, it is important to investigate whether this phenomenon still exists in a wideband reflectarray.

When a two-dimensional planar array with $15 \times 15$ elements is non-uniformly illuminated by a horn at $r_{f}$, as shown in Fig. 5 (a), the reradiated field from the array in an arbitrary direction $\vec{u}$ can be represented by

$$
\begin{gathered}
E(\vec{u})=\sum_{m=1}^{15} \sum_{n=1}^{15} F\left(\overrightarrow{r_{m n}} \cdot \overrightarrow{r_{f}}\right) A\left(\overrightarrow{r_{m n}} \cdot \overrightarrow{u_{0}}\right) A\left(\vec{u} \cdot \overrightarrow{u_{0}}\right) \\
\cdot e^{-j k_{0}\left(\left|\overrightarrow{r_{m n}}-\overrightarrow{r_{f}}\right|+\overrightarrow{r_{m n}} \cdot \vec{u}\right)} \cdot e^{j \phi_{m n}}
\end{gathered}
$$

where $F$ is the pattern function of the feed horn, $A$ is the pattern function of the S-shaped element, $\overrightarrow{r_{m n}}$ is the position vector of the $m n$th element, $\overrightarrow{u_{0}}$ is the desired main beam direction and $\phi_{m n}$ is the required phase delay of the $m n$th element $[10,24]$.

Considering an incident wave radiated by the feed horn propagating in the $-Z$ direction, as shown in Fig. 5 (a) $\vec{E}^{i n c}=F_{R H}\left(\overrightarrow{E_{x}}-j \overrightarrow{E_{y}}\right) e^{j k_{0} z} e^{j \omega \mathrm{t}}+F_{L H}\left(\overrightarrow{E_{x}}+j \overrightarrow{E_{y}}\right) e^{j k_{0} z} e^{j \omega \mathrm{t}}$

The former part represents the RHCP component of the radiated field from the horn with a magnitude of $F_{R H}$, the second part is the LHCP component with a magnitude of $F_{L H}$.

Denoting the physical counter-clockwise rotation angle of the $m n$th element by $\psi_{m n}$, the reflected RHCP field by the $m n$th $\mathrm{S}$-shaped element can be written in the form

$$
\begin{aligned}
\vec{E}_{R H C P}^{r e f l} & =\left|\Gamma_{\text {co-pol }}\right| \cdot F_{R H}\left(\overrightarrow{E_{x}}+j \overrightarrow{E_{y}}\right) e^{-j k_{0} z} e^{j \omega \mathrm{t}} \cdot e^{-2 j \psi_{m n}} \\
& +\left|\Gamma_{\text {cross }- \text { pol }}\right| \cdot F_{L H}\left(\overrightarrow{E_{x}}+j \overrightarrow{E_{y}}\right) e^{-j k_{0} z} e^{j \omega \mathrm{t}}
\end{aligned}
$$

The first part represents the reflected RHCP field from the incident RHCP component of the feed horn, and thus a counterclockwise rotation angle $\psi_{m n}$ of the element brings a $-2 \psi_{m n}$ phase variation to the reflected co-pol field. The second part denotes the reflected RHCP field from the incident LHCP component of the feed horn, and no additional phase variation is introduced upon reflection [10].

Similarly, the reflected LHCP field by the $m n$th S-shaped element with rotation angle of $\psi_{m n}$ can be represented by

$$
\begin{aligned}
\vec{E}_{L H C P}^{r e f l} & =\left|\Gamma_{\text {co-pol }}\right| \cdot F_{L H}\left(\overrightarrow{E_{x}}-j \overrightarrow{E_{y}}\right) e^{-j k_{0} z} e^{j \omega t} \cdot e^{2 j \psi_{m n}} \\
& +\left|\Gamma_{\text {cross-pol }}\right| \cdot F_{R H}\left(\overrightarrow{E_{x}}-j \overrightarrow{E_{y}}\right) e^{-j k_{0} z} e^{j \omega \mathrm{t}}
\end{aligned}
$$

It is worth pointing out that $\left|\Gamma_{\text {cross-pol }}\right|$ used in (3) and (4) is of the same value due to reciprocity and thus is denoted by the same expression. Considering that for a RHCP incident wave, the reflected co-pol component is RHCP field while for a LHCP incident wave, the reflected co-pol component is LHCP field, the magnitudes of the reflection coefficient $\left|\Gamma_{c o-p o l}\right|$ are the same in these two cases. However, the phases of the reflection coefficient $\angle \Gamma_{c o-p o l}$ in these two cases differ in signs, i.e. $\angle \Gamma_{c o-p o l}=-2 \psi_{m n}$ for a RHCP incident wave while 
$\angle \Gamma_{c o-p o l}=2 \psi_{m n}$ for a LHCP incident wave when the element is counter-clockwise rotated by an angle of $\psi_{m n}$.

From equation (1), the condition for the aperture distribution to be cophasal in the desired direction $\overrightarrow{u_{0}}$ is

$$
\phi_{m n}-k_{0}\left(\left|\overrightarrow{r_{m n}}-\overrightarrow{r_{f}}\right|+\overrightarrow{r_{m n}} \cdot \overrightarrow{u_{0}}\right)=2 k \pi, k=0,1,2 \cdots
$$

Here, the reflectarray is designed to radiate RHCP wave as its co-pol component. Thus, the co-pol component phase delay of the mnth element with a counter-clockwise rotation angle $\psi_{m n}$ under RHCP incident wave satisfies

$$
-2 \psi_{m n}-k_{0}\left(\left|\overrightarrow{r_{m n}}-\overrightarrow{r_{f}}\right|+\overrightarrow{r_{m n}} \cdot \overrightarrow{u_{0}}\right)=2 k \pi, k=0,1,2 \cdots \quad(6)
$$

Subsisting (3) and (6) into (1), the reradiated RHCP field in the desired direction $\overrightarrow{u_{0}}$ can be represented by

$$
\begin{gathered}
E_{R H C P}\left(\overrightarrow{u_{0}}\right)=\sum_{m=1}^{15} \sum_{n=1}^{15} A\left(\overrightarrow{r_{m n}} \cdot \overrightarrow{u_{0}}\right)\left\{\left|\Gamma_{c o-p o l}\right| \cdot F_{R H C P}\left(\overrightarrow{r_{m n}} \cdot \overrightarrow{r_{f}}\right)\right. \\
\left.\cdot e^{j 2 \mathrm{k \pi}}+\left|\Gamma_{\text {cross }- \text { pol }}\right| \cdot F_{L H C P}\left(\overrightarrow{r_{m n}} \cdot \overrightarrow{r_{f}}\right) \cdot e^{j 2 \mathrm{k} \pi} e^{j 2 \psi_{m n}}\right\}(7)
\end{gathered}
$$

Where $F_{R H C P}$ and $F_{L H C P}$ denote the pattern function of the feed horn in RHCP and LHCP, respectively. As aforementioned, the co-pol component phase delay of the $m n$th element under LHCP incident wave is $2 \psi_{m n}$. Therefore, the re-radiated LHCP field in the desired direction $\overrightarrow{u_{0}}$ can be written as

$$
\begin{aligned}
& E_{L H C P}\left(\overrightarrow{u_{0}}\right)=\sum_{m=1}^{15} \sum_{n=1}^{15} A\left(\overrightarrow{r_{m n}} \cdot \overrightarrow{u_{0}}\right)\left\{\left|\Gamma_{c o-p o l}\right| \cdot F_{L H C P}\left(\overrightarrow{r_{m n}} \cdot \overrightarrow{r_{f}}\right)\right. \\
& \left.\cdot e^{j 2 \mathrm{k} \pi} e^{j 4 \psi_{m n}}+\left|\Gamma_{\text {cross-pol }}\right| F_{R H C P}\left(\overrightarrow{r_{m n}} \cdot \overrightarrow{r_{f}}\right) e^{j 2 \mathrm{k} \pi} e^{j 2 \psi_{m n}}\right\}(8)
\end{aligned}
$$

For most of the elements, $\psi_{m n} \neq n \pi$, and it is evident that

$$
\begin{aligned}
& \left|\sum_{m=1}^{15} \sum_{n=1}^{15} e^{j 2 \psi_{m n}} e^{j 2 \mathrm{k} \pi}\right| \ll\left|\sum_{m=1}^{15} \sum_{n=1}^{15} e^{j 2 \mathrm{k} \pi}\right| \\
& \left|\sum_{m=1}^{15} \sum_{n=1}^{15} e^{j 4 \psi_{m n}} e^{j 2 \mathrm{k} \pi}\right| \ll\left|\sum_{m=1}^{15} \sum_{n=1}^{15} e^{j 2 \mathrm{k} \pi}\right|
\end{aligned}
$$

Since $\left|F_{R H C P}\left(\overrightarrow{r_{m n}} \cdot \overrightarrow{r_{f}}\right)\right|>\left|F_{L H C P}\left(\overrightarrow{r_{m n}} \cdot \overrightarrow{r_{f}}\right)\right|$ in the main beam region when the feed horn is RHCP and $\left|\Gamma_{\text {co-pol }}\right|>$ $\left|\Gamma_{\text {cross-pol }}\right|$ across the element's bandwidth, it can be concluded from equations (7)-(9) that

1) To design a RHCP reflectarray with good performance in terms of antenna gain, the CP purity of the feed should be high, i.e. the AR of the feed should be rather small. Otherwise, the incident LHCP field from the feed horn vanishes according to equations (7) and (8) since the reflection of the LHCP field does not superposes in-phase, which wastes the incident LHCP power from the feed and results in a low gain and poor AE. Meanwhile, the reflected cross-pol component of the element should also be low to keep the reflection of the incident RHCP field superposed in-phase. If $\left|\Gamma_{\text {cross-pol }}\right|$ is large, one part of the reflected field $\left|\Gamma_{\text {cross-pol }}\right| F_{R H C P}\left(\overrightarrow{r_{m n}} \cdot \overrightarrow{r_{f}}\right) e^{j 2 \mathrm{k} \pi} e^{j 2 \psi_{m n}}$ which does not superposes in-phase in the far-field, consumes the incident RHCP power substantially and also results in a decreased gain.

2) It is possible to get an enhanced $A R$ from the reflectarray even with a feed which has a large AR. Particularly considering a linearly polarized (LP) feed, the incident RHCP and LHCP power upon the aperture is the same. From equation (7), the reflected RHCP field can be greatly improved if $\left|\Gamma_{c o-p o l}\right|$ is close to 1 . Under the same condition, the reflected LHCP field is not superposed in-phase and thus is much smaller than the reflected RHCP field according to equation (8). Subsequently, the AR of the reflectarray is improved compared with the feed.
However, the gain and $\mathrm{AE}$ of the reflectarray is reduced in this case since the incident LHCP power contributes little to the total gain as analyzed in conclusion (1).

To verify the above analysis, the proposed reflectarray is illuminated by two different feed horns, one is a wideband dual$\mathrm{CP}$ horn with RHCP port excited and another one is an ideal RHCP horn which has infinite $\mathrm{CP}$ bandwidth $(\mathrm{AR}<3 \mathrm{~dB})$. Besides, the aperture size of these two horns are all kept $50 \mathrm{~mm}$ $\times 50 \mathrm{~mm}$ to give a similar gain and beamwidth at each frequency. When conducting the comparison, the two different feeds are placed at the same position to make the f/D unchanged. With these conditions, the spillover under these two cases are considered to be close to each other.

Fig. 7 shows the simulated AR of the proposed reflectarray with different feed horns.

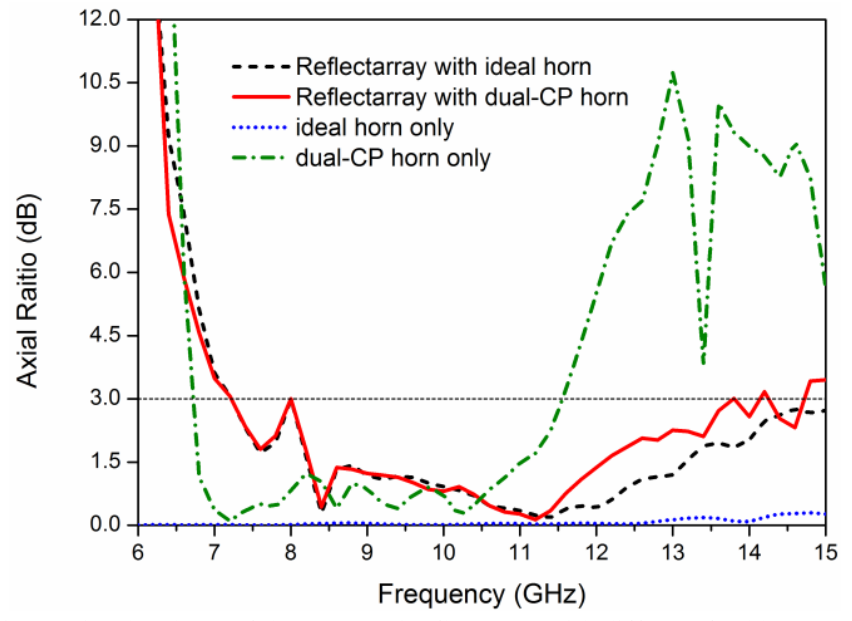

Fig. 7. Simulated AR of the proposed reflectarray with different feed horns.

As shown, the AR bandwidth $(\mathrm{AR}<3 \mathrm{~dB})$ of the dual-CP horn is from $6.7 \mathrm{GHz}$ to $11.5 \mathrm{GHz}$ and the $\mathrm{AR}$ bandwidth of the reflectarray using the dual-CP horn is from $7.2 \mathrm{GHz}$ to 14.7GHz. From $6.7 \mathrm{GHz}$ to $7.2 \mathrm{GHz}$, the AR gets degraded from the horn to the array since the element reflection performance is rather bad in this frequency range $\left(\left|\Gamma_{\text {cross-pol }}\right|\right.$ approaches to $-5 \mathrm{~dB}$ at $6.7 \mathrm{GHz}$ as shown in Fig. 2). However, from $11.5 \mathrm{GHz}$ to $14.7 \mathrm{GHz}$, the AR of the array gets improved although the dual-CP horn demonstrates poor $\mathrm{CP}$ purity in this frequency range. This mainly attributes to the function of the array as indicated in the above conclusion (2) and the relative good element reflection performance $\left(\left|\Gamma_{\text {cross-pol }}\right|=-10.2 \mathrm{~dB}\right.$, $\left|\Gamma_{c o-p o l}\right|=-0.48 \mathrm{~dB}$ at $\left.14.7 \mathrm{GHz}\right)$. It is also shown in Fig. 7 that better AR performance can be realized with feed of better $\mathrm{CP}$ purity (referring to the AR of the reflectarray with ideal horn and dual-CP horn, respectively, from $11.5 \mathrm{GHz}$ to $15 \mathrm{GHz}$ ).

Fig. 8 shows the simulated gain of the proposed reflectarray with different feed horns. As shown, the gain of the reflectarray with dual-CP horn is smaller than the array with ideal horn from $11.5 \mathrm{GHz}$ to $15 \mathrm{GHz}$. In this frequency range, the gain of the two horns are generally the same, especially from $13.5 \mathrm{GHz}$ to 14.5GHz. As analyzed in the aforementioned conclusion (1), the poor $\mathrm{CP}$ purity of the dual-CP horn in this frequency range results in the non-negligible LHCP incident field which contributes little to the gain of the array and yields a lower gain 
compared with the array using an ideal horn which has a much better $\mathrm{CP}$ purity in the same frequency range.

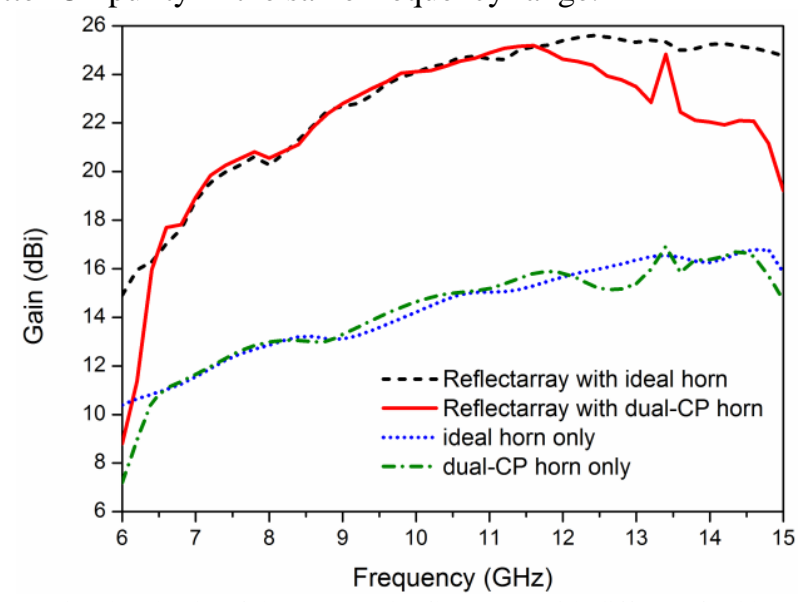

Fig. 8. Simulated gain of the proposed reflectarray with different feed horns.

\section{Effect of Differential Spatial Phase Delay}

As indicated in last section, the proposed reflectarray achieves a 2:1 3-dB AR bandwidth. Due to this broad bandwidth, it is necessary to investigate the influence of the differential spatial phase delay to the gain bandwidth of the array, which increases as the working bandwidth increases. The bandwidth limitation of printed reflectarrays caused by the differential spatial phase delay is quantified for a broadside main beam and a prime focus feed in [25]. Here, equations are derived for more general cases, i.e. arbitrary feed position and arbitrary main beam direction to quantify the bandwidth limitation of reflectarrays due to the differential spatial phase delay.

Fig. 9 shows the geometry of a reflectarray with arbitrary feed position and arbitrary main beam direction. As shown, the diameter of the reflectarray is denoted by $\mathrm{D}$ while the focal length $f=\sqrt{r^{2}+h^{2}}$. The feed horn is placed at an arbitrary position with an incident angle $\theta_{i}$ to the aperture center $\mathrm{O}$ and the main beam direction is denoted by $\theta_{r}$.

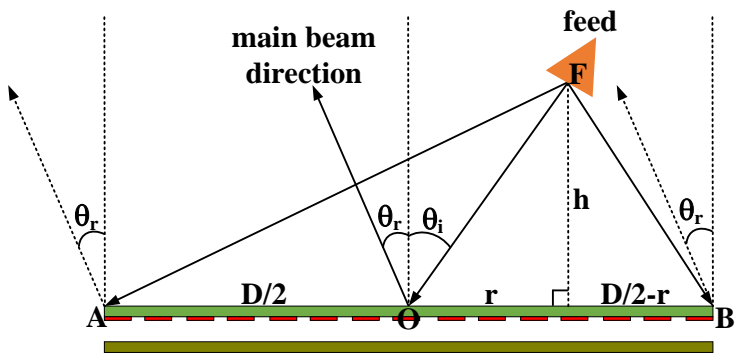

Fig. 9. Geometry of the reflectarray with arbitrary feed position and arbitrary main beam direction.

Denoting the spatial phase delay from the feed to the aperture center $\mathrm{O}$ by $P D_{F O}$ and the spatial phase delay from the feed to the edge of the aperture by $P D_{F A}$ and $P D_{F B}$. As the main beam direction is $\theta_{r}$, the additional spatial phase delay introduced by the offset beam between the aperture center and the edge of the aperture is $\frac{D}{2} \cdot \sin \theta_{r}$. Therefore, the phase delay required by the aperture edge element $\mathrm{A}$ and $\mathrm{B}$ with respect to the aperture center $\mathrm{O}$ can be represented by $P D_{A O}$ and $P D_{B O}$, which satisfy

$$
\begin{gathered}
P D_{A O}=P D_{F A}-P D_{F O}-D \cdot \sin \theta_{r} / 2 \\
P D_{B O}=P D_{F B}-P D_{F O}+D \cdot \sin \theta_{r} / 2
\end{gathered}
$$

Let $k_{0}$ and $k_{1}$ be the wavenumbers at the design frequency $f_{0}$ and operating frequency $f_{1}$, respectively. When the frequency shifts from the design frequency $f_{0}$ to the operating frequency $f_{1}$, phase errors will be introduced to each element. Denote the phase error at the edge element $\mathrm{A}$ and $\mathrm{B}$, relative to the phase at the center element, by $P E_{A O}$ and $P E_{B O}$, which are

$$
\begin{aligned}
& P E_{A O}=\left(k_{1}-k_{0}\right) R_{A} \\
& P E_{B O}=\left(k_{1}-k_{0}\right) R_{B}
\end{aligned}
$$

where $R_{A}=\left(\sqrt{(D / 2+r)^{2}+h^{2}}-\sqrt{r^{2}+h^{2}}-D \cdot \sin \theta_{r} / 2\right)$ and $R_{B}=$ $\left(\sqrt{(D / 2-r)^{2}+h^{2}}-\sqrt{r^{2}+h^{2}}+D \cdot \sin \theta_{r} / 2\right)$, calculating from the geometry relationship shown in Fig. 9. According to [25], a reasonable criterion for evaluating the effect of this phase error is to evaluate the frequency shift for which this error equals $180^{\circ}$ at the edge of the aperture. The intervening elements will incur less phase error, but out-of-phase radiation from edge elements $\mathrm{A}$ and $\mathrm{B}$ will begin to detract from the overall gain of the aperture. Let $P E_{A O}$ and $P E_{B O}$ equal to $180^{\circ}$ and solve for the frequency shift, $\Delta f=f_{1}-f_{0}$, we can get:

$$
\frac{\Delta f}{f_{0}}=\frac{\lambda_{0}}{2 R_{A}} \text {, for } P E_{A O}=\pi
$$$$
\frac{\Delta f}{f_{0}}=\frac{\lambda_{0}}{2 R_{B}} \text {, for } P E_{B O}=\pi \quad \text { (12)Substituting }
$$

geometry dimensions $\mathrm{D}, \mathrm{r}, \mathrm{h}$ and $\theta_{r}$ given in Fig. 5 (a) to equation (12), the frequency bandwidth $2 \Delta f / f_{0}$ is calculated to be $200 \%$ and $145.5 \%$ respectively for $P E_{A O}=\pi$ and $P E_{B O}=\pi$ (the design frequency $f_{0}$ is $9 \mathrm{GHz}$ ). This result indicates that the gain of the proposed reflectarray suffers very limited degradation due to the differential spatial phase delay within a $145.5 \%$ bandwidth. As this calculated bandwidth is much bigger than the element bandwidth, the bandwidth of the proposed reflectarray is mainly determined by the element bandwidth.

\section{RESULTS AND DISCUSSIONS}

\section{A. Prototype and Reflection Coefficient}

The fabricated prototype of the proposed antenna and the measurement setup in the anechoic chamber are shown in Fig. 10. As shown, the reflectarray is fed by a wideband dual-CP horn which uses a stepped-septum polarizer to simultaneously achieve $\mathrm{TE}_{10}$ and $\mathrm{TE}_{01}$ modes as well as a 90-degree phase shift between the two orthogonal electric field components [26]. 


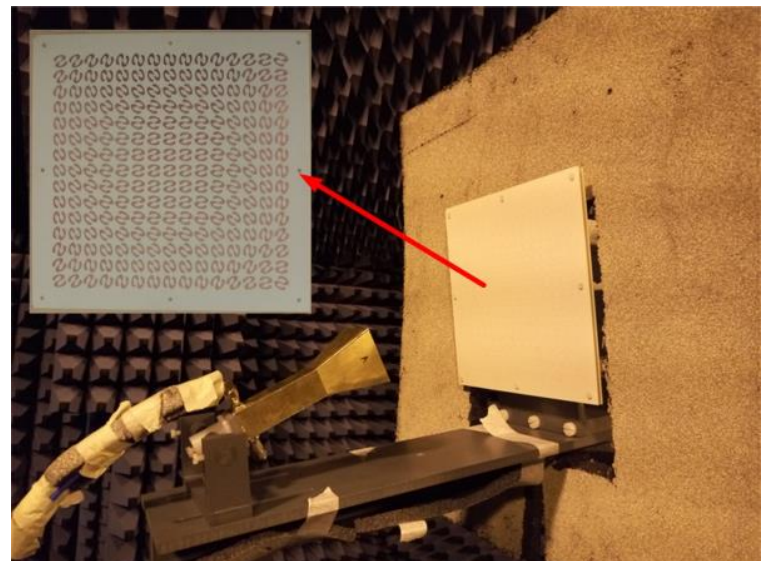

Fig. 10. The fabricated prototype and the measurement setup of the proposed antenna.

The simulated and measured reflection coefficients of the proposed reflectarray are shown in Fig. 11. As shown, the array achieves a smaller than $-10 \mathrm{~dB}$ reflection coefficient over a 2:1 frequency range. It is worthy pointing out that the $S_{11}$ will degrade when a prime focus feed is utilized due to the blockage of the feed horn. To eliminate this phenomenon, an offset feed is chosen.

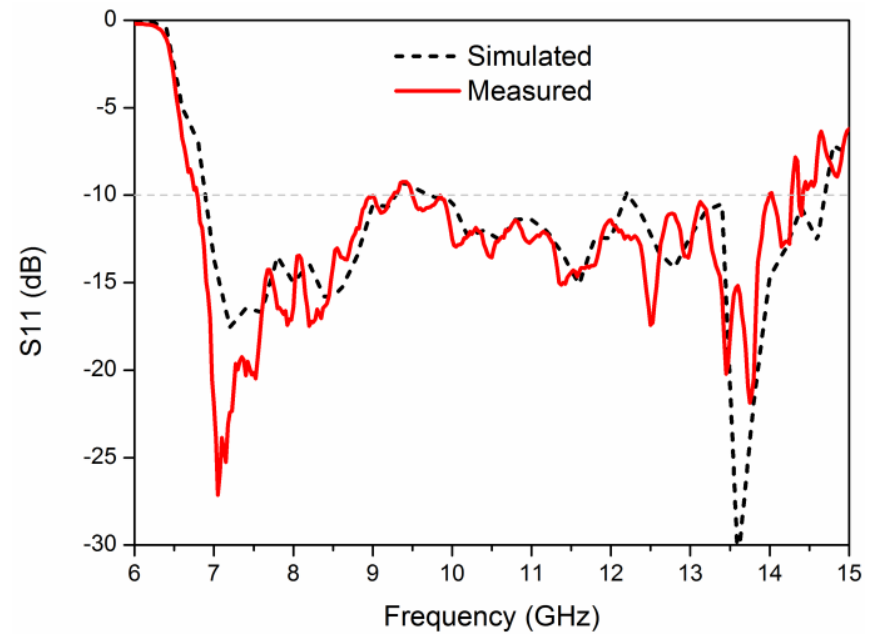

Fig. 11. Simulated and measured reflection coefficients of the proposed reflectarray.

\section{B. Axial Ratio}

The simulated and measured AR of the proposed antenna is shown in Fig. 12. As shown, the measured 3-dB AR bandwidth is from $7 \mathrm{GHz}$ to $14.3 \mathrm{GHz}$, equaling to a fractional bandwidth of $68.5 \%$.

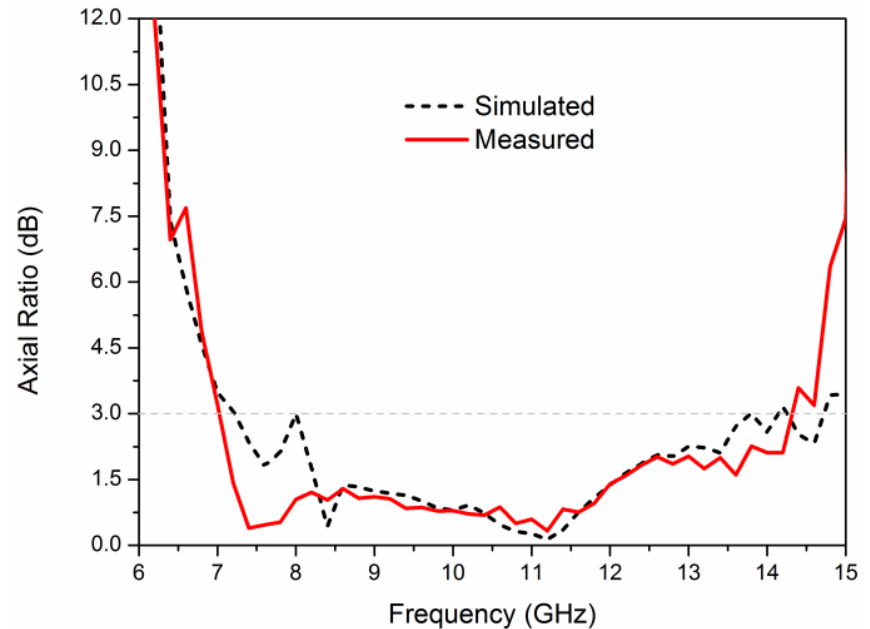

Fig. 12. Simulated and measured AR of the proposed antenna.

\section{Realized Gain and Aperture Efficiency}

Fig. 13 gives the simulated realized gain and measured gain of the proposed reflectarray. It is shown that the realized gain decreases rapidly when the frequency is lower than $7 \mathrm{GHz}$. This is mainly caused by the drastic deterioration of the horn's reflection coefficient when the frequency exceeds the working frequency range of the horn, as shown in Fig. 11. As shown in Fig. 13, the measured $3-\mathrm{dB}$ gain variation bandwidth is from $8.6 \mathrm{GHz}$ to $14 \mathrm{GHz}(47.8 \%)$. The measured gain peaks at $11.4 \mathrm{GHz}$ with a value of $25.25 \mathrm{dBic}$.

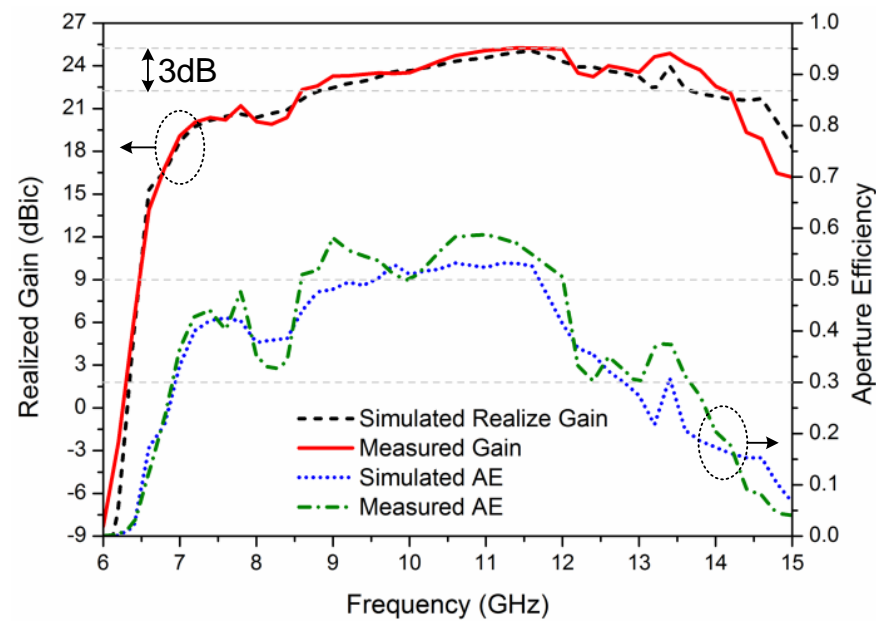

Fig. 13. Simulated and measured gain and efficiency of the proposed antenna.

The aperture efficiency is also given by Fig. 13. The simulated AE is calculated based on the simulated realized gain while the measured AE is calculated by the measured gain. The difference between the results may stem from the fabrication errors of the horn and the array as well as the measurement errors. From the measured result, the reflectarray can achieve larger than $50 \% \mathrm{AE}$ from $8.6 \mathrm{GHz}$ to $12 \mathrm{GHz}(33 \%)$ and larger than $30 \%$ AE from $7 \mathrm{GHz}$ to $13.6 \mathrm{GHz}(64 \%)$.

\section{Radiation Pattern}

As aforementioned, the proposed reflectarray achieves a nearly $2: 1$ bandwidth in terms of impedance bandwidth, $3 \mathrm{~dB}$ AR bandwidth and 30\% AE bandwidth. Moreover, the 
proposed reflectarray can also maintain good radiation pattern performance such as undistorted radiation pattern shape, low sidelobe and low cross-pol component in a 2:1 bandwidth. To verify this, the radiation patterns at five different frequencies are shown in Fig. 14. The E-plane in Fig. 14 refers to the xoz plane as shown in Fig. 5 while the H-plane is the plane perpendicular to E-plane along the main beam direction.
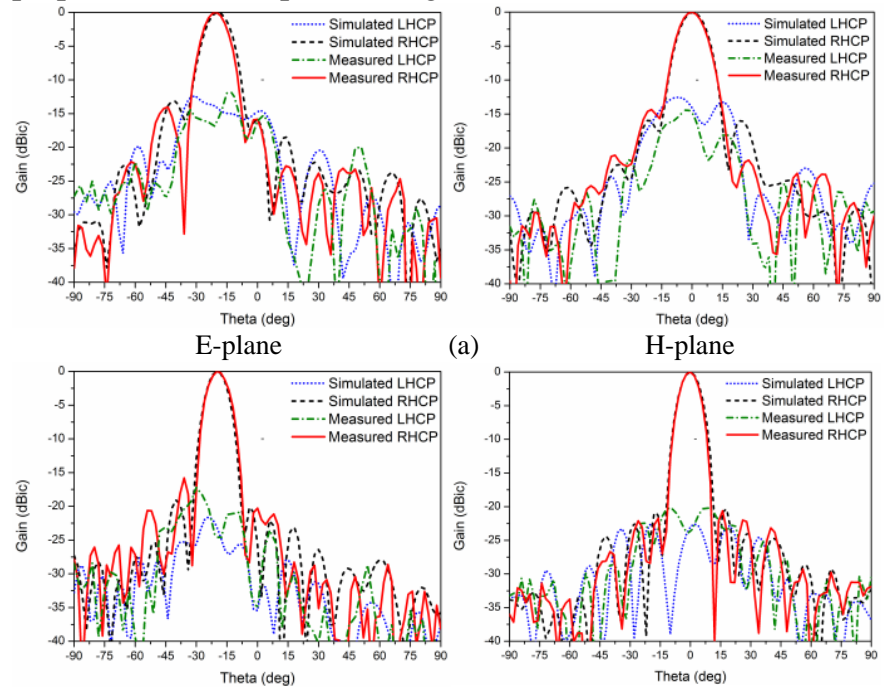

(a)

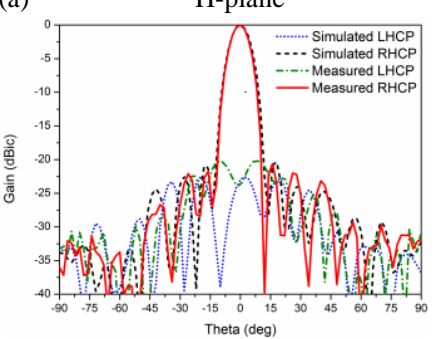
E-plane

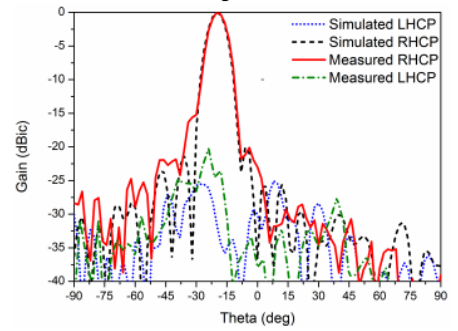

(b)

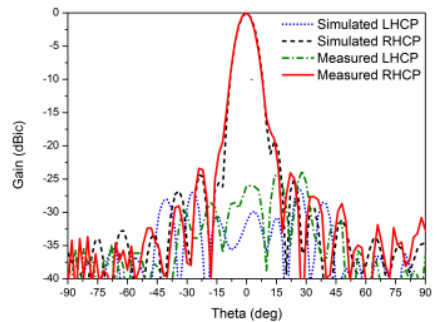

(c)
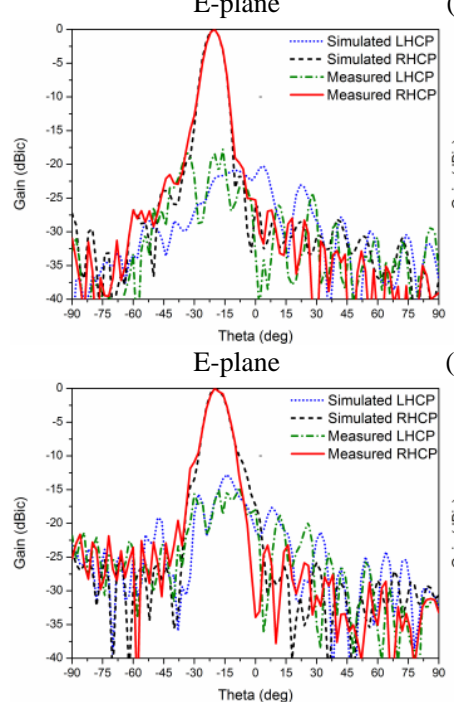

E-plane

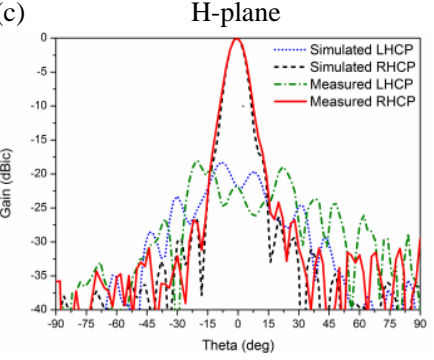

(d)

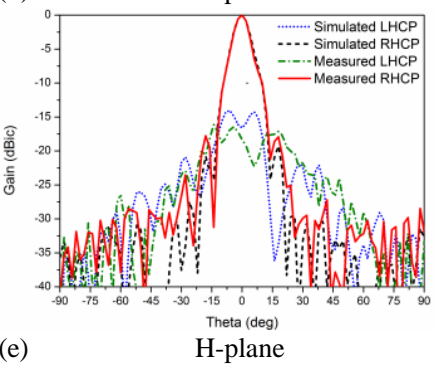

Fig. 14. Simulated and measured radiation patterns of the proposed antenna: (a) $7 \mathrm{GHz}$, (b) $9 \mathrm{GHz}$, (c) $10.6 \mathrm{GHz}$, (d) $12 \mathrm{GHz}$, (e) $14 \mathrm{GHz}$.

As shown in Fig. 14, the measured radiation pattern agrees well with the simulated radiation pattern at each frequency. It is also observed from the results that the array maintains undistorted pencil-shaped beams, at least $-15 \mathrm{~dB}$ side lobe and -

$15 \mathrm{~dB}$ cross-pol component from $7 \mathrm{GHz}$ to $14 \mathrm{GHz}$.

\section{E. Comparison with Other Wideband CP Reflectarrays}

Due to the wideband characteristic of the novel S-shaped phasing element, the presented reflectarray achieves wide bandwidth in all aspects. To demonstrate its advantages more clearly, a comparison between the proposed design and other reported wideband CP reflectarrays is given by Table II. As shown in the table, the presented design provides wider bandwidth (BW) in terms of 3-dB AR BW, 3-dB gain BW and radiation pattern $\mathrm{BW}$. More importantly, the proposed reflectarray can maintain higher than $50 \%$ aperture efficiency in a 33\% bandwidth, which is rather difficult to achieve.

TABLE II

COMPARISON WITH OTHER WIDEBAND CP REFLECTARRAYS

\begin{tabular}{|c|c|c|c|c|c|}
\hline $\begin{array}{l}\text { Ref. } \\
\text { No. }\end{array}$ & Layers & $\begin{array}{c}3-\mathrm{dB} \\
\text { AR BW } \\
\end{array}$ & Gain BW & AE BW & $\begin{array}{c}\text { Radiation } \\
\text { Pattern BW }\end{array}$ \\
\hline [9] & Single & $50 \%$ & $\begin{array}{c}\text { 1-dB gain } \\
12.5 \%\end{array}$ & - & - \\
\hline [17] & Single & $11 \%$ & $\begin{array}{c}\text { 1-dB gain } \\
17 \%\end{array}$ & - & - \\
\hline [18] & Dual & $28 \%$ & $\begin{array}{c}1 \text {-dB gain } \\
20 \%\end{array}$ & - & - \\
\hline [19] & Single & $43 \%$ & $\begin{array}{c}\text { 1-dB gain } \\
31 \% \\
3-\mathrm{dB} \text { gain } \\
\sim 40 \%\end{array}$ & - & - \\
\hline [20] & Multiple & $40 \%$ & $\begin{array}{c}\text { 3-dB gain } \\
40 \%\end{array}$ & - & $40 \%$ \\
\hline $\begin{array}{l}\text { This } \\
\text { work }\end{array}$ & Single & $68.5 \%$ & $\begin{array}{c}\text { 3-dB gain } \\
47.8 \%\end{array}$ & $\begin{array}{l}>50 \%: \\
33 \% \\
>30 \%: \\
64 \%\end{array}$ & $66.7 \%$ \\
\hline
\end{tabular}

\section{CONCLUSION}

A single layer wideband circularly polarized reflectarray using a novel S-shaped phasing element was presented. The proposed S-shaped element achieves low cross-pol reflection and very linear phase shift to the element rotation angle in a 2:1 bandwidth. Theoretical analysis is given to indicate that the AR bandwidth of the $\mathrm{CP}$ reflectarray can be improved using element rotation method even with incident fields poor in $\mathrm{CP}$ purity. Moreover, the influence of differential spatial phase delay on the bandwidth of the proposed array is proved to be very limited. The measurement results demonstrate that the proposed reflectarray has a $68.5 \% 3 \mathrm{~dB}$ AR bandwidth and a $47.8 \% 3 \mathrm{~dB}$ gain bandwidth. More importantly, the aperture efficiency of the proposed array is larger than $50 \%$ in a $33 \%$ bandwidth and larger than $30 \%$ in a $64 \%$ bandwidth. These excellent performances make the proposed reflectarray a good candidate for high-data rate satellite communications.

\section{REFERENCES}

[1] J. Huang and J. A. Encinar, Reflectarray Antennas: John Wiley \& Sons, 2007.

[2] J. A. Encinar and J. A. Zornoza, "Broadband design of three-layer printed reflectarrays," IEEE Trans. Antennas Propag., vol. 51, pp. 1662-1664, 2003. 
[3] J. A. Encinar, "Design of two-layer printed reflectarrays using patches of variable size," IEEE Trans. Antennas Propag., vol. 49, pp. 1403-1410, 2001.

[4] P.-Y. Qin, Y. J. Guo, and A. R. Weily, "Broadband Reflectarray Antenna Using Subwavelength Elements Based on Double Square Meander-Line Rings," IEEE Trans. Antennas Propag., vol. 64, pp. 378-383, 2016.

[5] Y. Mao, S. Xu, F. Yang, and A. Z. Elsherbeni, "A novel phase synthesis approach for wideband reflectarray design," IEEE Trans. Antennas Propag., vol. 63, pp. 4189-4193, 2015.

[6] E. Carrasco, J. A. Encinar, and M. Barba, "Bandwidth improvement in large reflectarrays by using true-time delay," IEEE Trans. Antennas Propag., vol. 56, pp. 2496-2503, 2008.

[7] S. Gao, Q. Luo, and F. Zhu, Circularly polarized antennas: John Wiley \& Sons, 2013.

[8] Y. Li, M. E. Bialkowski, and A. M. Abbosh, "Single layer reflectarray with circular rings and open-circuited stubs for wideband operation," IEEE Trans. Antennas Propag., vol. 60, pp. 4183-4189, 2012.

[9] G.-B. Wu, S.-W. Qu, S. Yang, and C. H. Chan, "Broadband, Single-Layer Dual Circularly Polarized Reflectarrays With Linearly Polarized Feed," IEEE Trans. Antennas Propag., vol. 64, pp. 4235-4241, 2016.

[10] J. Huang and R. J. Pogorzelski, "A Ka-band microstrip reflectarray with elements having variable rotation angles," IEEE Trans. Antennas Propag., vol. 46, pp. 650-656, 1998.

[11] A. Yu, F. Yang, A. Elsherbeni, J. Huang, and Y. Kim, "An offset-fed Xband reflectarray antenna using a modified element rotation technique," IEEE Trans. Antennas Propag., vol. 60, pp. 1619-1624, 2012.

[12] R. Deng, Y. Mao, S. Xu, and F. Yang, "A Single-Layer Dual-Band Circularly Polarized Reflectarray With High Aperture Efficiency," IEEE Trans. Antennas Propag., vol. 63, pp. 3317-3320, 2015.

[13] R. S. Malfajani and Z. Atlasbaf, "Design and implementation of a dualband single layer reflectarray in $\mathrm{X}$ and $\mathrm{K}$ bands," IEEE Trans. Antennas Propag., vol. 62, pp. 4425-4431, 2014.

[14] C. Han, J. Huang, and K. Chang, "A high efficiency offset-fed X/Ka-dualband reflectarray using thin membranes," IEEE Trans. Antennas Propag., vol. 53, pp. 2792-2798, 2005.

[15] T. Smith, U. Gothelf, O. S. Kim, and O. Breinbjerg, "An FSS-backed $20 / 30 \mathrm{GHz}$ circularly polarized reflectarray for a shared aperture L-and Ka-band satellite communication antenna," IEEE Trans. Antennas Propag., vol. 62, pp. 661-668, 2014.

[16] W. A. Imbriale, S. Gao, and L. Boccia, Space antenna handbook: Wiley, 2012.

[17] G. Zhao, Y.-C. Jiao, F. Zhang, and F.-S. Zhang, "A subwavelength element for broadband circularly polarized reflectarrays," IEEE Antennas Wireless Propag. Lett., vol. 9, pp. 330-333, 2010.

[18] L.-S. Ren, Y.-C. Jiao, F. Li, J.-J. Zhao, and G. Zhao, "A dual-layer Tshaped element for broadband circularly polarized reflectarray with linearly polarized feed," IEEE Antennas Wireless Propag. Lett., vol. 10, pp. 407-410, 2011.

[19] M.-Y. Zhao, G.-Q. Zhang, X. Lei, J.-M. Wu, and J.-Y. Shang, "Design of new single-layer multiple-resonance broadband circularly polarized reflectarrays," IEEE Antennas Wireless Propag. Lett., vol. 12, pp. 356$359,2013$.

[20] S. M. A. M. H. Abadi and N. Behdad, "Broadband True-Time-Delay Circularly-Polarized Reflectarray With Linearly-Polarized Feed," IEEE Trans. Antennas Propag., in press.

[21] L. Zhang, S. Gao, Q. Luo, P. R. Young, L. Wenting, and Q. Li, "InvertedS Antenna with Wideband Circular Polarization and Wide Axial Ratio Beamwidth," IEEE Trans. Antennas Propag., in press.

[22] A. Mahmoud, A. A. Kishk, Z. Hao, and W. Hong, "Ka-band circularly polarized reflectarray: Using a double-layers cross slot," IEEE Antennas Propag. Mag., vol. 58, pp. 60-68, 2016.

[23] D. M. Pozar, S. D. Targonski, and H. Syrigos, "Design of millimeter wave microstrip reflectarrays," IEEE Trans. Antennas Propag., vol. 45, pp. $287-$ 296, 1997.

[24] P. Nayeri, A. Z. Elsherbeni, and F. Yang, "Radiation Analysis Approaches for Reflectarray Antennas," IEEE Antennas Propag. Mag., vol. 55, pp. 127-134, 2013.

[25] D. M. Pozar, "Bandwidth of reflectarrays," Electron. Lett., vol. 39, pp. 1490-1491, 2003.

[26] J. Bornemann and V. A. Labay, "Ridge waveguide polarizer with finite and stepped-thickness septum," IEEE Trans. Microw. Theory Tech., vol. 43, pp. 1782-1787, 1995. 